\title{
Mixed-state microwave response in superconducting cuprates.
}

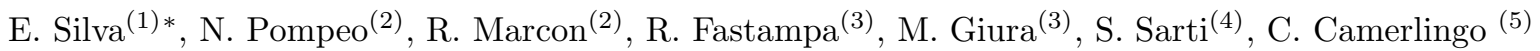 \\ (1) Dipartimento di Fisica "E. Amaldi" and INFM-Coherentia, Università di Roma Tre, Via della Vasca Navale 84, 00146 \\ Roma, Italy \\ ${ }^{(2)}$ Dipartimento di Fisica "E. Amaldi" and INFM, Università di Roma Tre, 00146 Roma, Italy \\ (3) Dipartimento di Fisica and INFM-Coherentia, Università "La Sapienza", 00185 Roma, Italy \\ (4) Dipartimento di Fisica and INFM, Università "La Sapienza", 00185 Roma, Italy \\ (5) CNR-Istituto di Cibernetica "E. Caianiello", 80078 Pozzuoli, Napoli, Italy
}

(submitted June 15th, 2004)

\begin{abstract}
We report measurements of the magnetic-field induced microwave complex resistivity in $\mathrm{REBa}_{2} \mathrm{Cu}_{3} \mathrm{O}_{7-\delta}$ thin films, with $\mathrm{RE}=\mathrm{Y}, \mathrm{Sm}$. Measurements are performed at $48 \mathrm{GHz}$ by means of a resonant cavity in the end-wall-replacement configuration. The magnetic field dependence is investigated by applying a moderate $(0.8 \mathrm{~T})$ magnetic field along the $c$-axis. The measured vortex state complex resistivity in $\mathrm{YBa}_{2} \mathrm{Cu}_{3} \mathrm{O}_{7-\delta}$ and $\mathrm{SmBa}_{2} \mathrm{Cu}_{3} \mathrm{O}_{7-\delta}$ is analyzed within the well-known models for vortex dynamics. It is shown that attributing the observed response to vortex motion alone leads to inconsistencies in the as-determined vortex parameters (such as the vortex viscosity and the pinning constant). By contrast, attributing the entire response to field-induced pair breaking leads to a nearly quantitative description of the data.
\end{abstract}

KEYWORDS: microwave resistivity, vortex state, pair breaking

\section{INTRODUCTION}

The microwave response is a source of important information in superconductors. In high- $T_{c}$ superconductors (HTCS) various microwave techniques have been used to get information, among the others, on the symmetry of the order parameter, on the vortex parameters such as the vortex viscosity and pinning frequency and on the temperature dependence of the superfluid fraction (via the measurement of the temperature dependence of the London penetration depth). In short, microwave measurements are a very powerful tool to study the superconducting state.

While $\mathrm{YBa}_{2} \mathrm{Cu}_{3} \mathrm{O}_{7-\delta}$ (YBCO) and $\mathrm{Bi}_{2} \mathrm{Sr}_{2} \mathrm{CaCu}_{2} \mathrm{O}_{8+x}$ (BSCCO) have been the subject of intensive experimental investigation at microwave frequencies, other HTCS did not receive the same attention. In particular, a very few reports dealt with the vortex-state microwave response in rare-earth substituted 123 compounds, mainly on Gd-substituted materials ${ }^{1}$. In fact, RE-substituted 123 compounds present interesting features in the vortex state: in particular, they often give enhanced irreversibility lines and in some cases a slightly higher $T_{c}$. Due to their very similarity to YBCO, they are not thought to present novel features in the basic mechanisms governing the superconducting properties. Aim of this paper is to present a compared study of the microwave response of $\mathrm{YBCO}$ and $\mathrm{SmBa}_{2} \mathrm{Cu}_{3} \mathrm{O}_{7-\delta}(\mathrm{SmBCO})$ in the vortex state in moderate fields. It will be shown that some quantitative and qualitative differences exist between the field dependence of the complex resistivity in the two materi- als. However, the analysis of the data shows that those differences cannot be related only to changes in the vortex dynamics (e.g., different pinning). Indeed, a close analysis of the data shows that vortex motion cannot be the main contribution to the observed resistivity in any of the two materials. Rather, we find that the resistivity of both YBCO and SmBCO can be qualitatively described assuming that the response is mainly determined by magnetic field induced pair breaking. Within this frame, the differences between the two materials can be ascribed to different quasiparticle scattering time below $T_{c}$.

We recall the main phenomena lying at the base of the microwave response in the vortex state. In principle, the response is dictated by vortex motion, described by the vortex motion complex resistivity $\tilde{\rho}_{v m}$, and by charge carriers complex conductivity, $\tilde{\sigma}$. The latter originates from superfluid as well as normal carriers.

A general frame that includes those contributions is the Coffey-Clem theory ${ }^{2}$. It is noteworthy that the theory, while developed for a particular case of vortex motion (vortex in a sinusoidal potential submitted to the Lorentz force and to a stochastic force), is not constrained to a specific potential shape seen by the vortices. Given the vortex motion resistivity $\tilde{\rho}_{v m}$ and the charge carriers complex conductivity $\tilde{\sigma}=\sigma_{1}-\mathrm{i} \sigma_{2}$, the resulting expression for the total complex resistivity can be written as:

$$
\tilde{\rho}=\frac{\tilde{\rho}_{v m}+\frac{\mathrm{i}}{\sigma_{2}}}{1+\mathrm{i} \frac{\sigma_{1}}{\sigma_{2}}}
$$

When the field dependence of the pair breaking (affecting $\tilde{\sigma})$ is negligible, and for $T$ sufficiently below $T_{c}$ such

*To whom correspondence should be addressed. E-mail: silva@fis.uniroma3.it 
that $\sigma_{2} \gg \sigma_{1}$, one has for the field induced change of the resistivity:

$$
\Delta \tilde{\rho}=\tilde{\rho}(H, T)-\tilde{\rho}(0, T)=\tilde{\rho}_{v m}(H, T)
$$

since the vortex motion resistivity is zero by definition in zero field. By contrast, when the vortex motion resistivity is vanishingly small, equation 1 reduces to:

$$
\tilde{\rho}(H, T)=\frac{1}{\tilde{\sigma}(H, T)}
$$

The vortex motion approximation alone has been often used for the analysis of the microwave response in the vortex state ${ }^{3}$ sometimes including a field-independent contribution from the superfluid. ${ }^{4-6}$ In this case, from the data one can estimate vortex parameters such as the vortex viscosity, the depinning frequency and the pinning constant and the activation energy. By contrast, it has been shown that in BSCCO films the imaginary conductivity could be well described ${ }^{7}$ by expression 3 . In this latter case characteristic parameters determining the response are the quasiparticle scattering time and the pair-breaking field.

\section{EXPERIMENTAL SECTION}

The $\mathrm{SmBa}_{2} \mathrm{Cu}_{3} \mathrm{O}_{7}$ film was grown on $1 \mathrm{~cm} \times 1 \mathrm{~cm}$ $\mathrm{LaAlO}_{3}$ substrate by planar high oxygen pressure dc sputtering technique. The roughness of $\sim 20 \mathrm{~nm}$ on $1 \mu \mathrm{m}$ $\times 1 \mu \mathrm{m}$ area was measured by AFM. The $\mathrm{YBa}_{2} \mathrm{Cu}_{3} \mathrm{O}_{7}$ film was grown by inverted cylindrical magnetron sputtering on $1 \mathrm{~cm} \times 1 \mathrm{~cm} \mathrm{CeO}$ buffered YSZ substrates. In both samples, out-of-plane c-axis orientation was assessed by rocking curves, that showed $\mathrm{FWHM} \approx 0.2^{\circ}$. Film thickness was $d \sim 200 \mathrm{~nm}$. $\Phi$-scan showed good epitaxiality. YBCO and SmBCO showed $T_{c} \simeq 88 \mathrm{~K}$ and 87 $\mathrm{K}$, respectively. Further details on sample preparation have been reported elsewhere. ${ }^{8,9}$

The microwave response was measured by the end-wall cavity technique at $48.2 \mathrm{GHz}$ in zero dc magnetic field, and with an applied field (along the $c$-axis) $\mu_{0} H \leq 0.8 \mathrm{~T}$. The cylindrical cavity resonated in the $\mathrm{TE}_{011}$ mode. ${ }^{10}$ In this configuration the microwave field probed a circular area, corresponding to the diameter of the cavity, $\sim 8 \mathrm{~mm}$, centered on the center of the film. We note that in this configuration the edges of the film are not exposed to the microwave field, so that our measurements are not affected by detrimentals edge effects (unlike, e.g., dc and microwave measurements on striplines). Due to the field distribution of the excited mode, the peak microwave field $\mu_{0} H_{\mu w} \sim 10 \mu \mathrm{T}$ is reached in an annular area of $4 \mathrm{~mm}$ diameter centered on the center of the film. Thus, for all practical purposes, the amplitude of the microwave field $H_{\mu w} \ll H$, so all the field effect should be regarded as coming from the dc applied field. Field-induced variations of the quality factor $\Delta \frac{1}{Q}$ and of the resonance frequency $\Delta \nu$ were measured at several temperatures in the range from $67(69)$ to $90 \mathrm{~K}$, resulting in reduced temperature ranges above $T / T_{c}=0.76$ $(0.79)$ in YBCO (SmBCO). Data have been collected either in zero-field-cooled, field-cooled, and on direct and reverse field-sweeping. We observed no hysteresis, with the possible exception of the data below $\sim 70 \mathrm{~K}$, where a small hysteresis, smaller than $10 \%$ of the overall signal is occasionally present on reverse field sweeps. In this case, data for field sweeps after ZFC are considered. We note that rather high operating temperatures and the strong demagnetization present in our arrangement (the dc field is perpendicular to the square film) lead to a very small value of the first penetration field, and in particular to $B \simeq \mu_{0} H$ even at low fields. ${ }^{11}$ However, for conservative reasons, we will not focus the discussion on the low field data (below $10 \mathrm{mT}$ ). Figure 1 reports the temperature variations of the cavity parameters reflecting the superconducting transition in zero magnetic field in YBCO (left panel) and SmBCO (right panel). As can be seen, a substantial background is present in the measurement of the frequency shift, indicating relevant mechanical relaxations of the metallic resonator. Even if the background was rather reproducible, we have chosen to work with field variations at fixed temperatures. In this case, the variation of the effective impedance does not depend on the background (but only on a geometrical factor $G$, which acts as a scale factor), and $\Delta \frac{1}{Q}$ and $\Delta \nu$ directly yield the field induced changes of the effective surface impedance of the thin film, according to: $G\left[\Delta \frac{1}{Q}-\mathrm{i} 2 \frac{\Delta \nu}{\nu_{0}}\right]=\Delta Z_{s}^{\text {eff }}(H) \simeq[\Delta \tilde{\rho}(H)] / d=$ $\left[\Delta \rho_{1}(H)+\mathrm{i} \Delta \rho_{2}(\mathrm{H})\right] / d$, where $\tilde{\rho}$ is the complex resistivity. Here, we have made use of the thin film approximation, extensively examined in Ref. 12 .

In figure 2 we show the effect of the application of a $0.7 \mathrm{~T}$ magnetic field in YBCO and SmBCO, respectively. The field-induced dissipation has a similar behaviour in both materials: $\left[\rho_{1}(T, 0.7 \mathrm{~T})-\rho_{1}(\mathrm{~T}, 0)\right] / d$ increases with increasing temperature, and then vanishes at the transition temperature, as qualitatively expected. The variation of the imaginary part $\left[\rho_{2}(T, 0.7 \mathrm{~T})-\rho_{2}(\mathrm{~T}, 0)\right] / d$ exhibits a quantitatively different behaviour in the two materials: in YBCO it is positive but small, apart the region very close to the transition where, at a temperature $T_{0}$, it becomes negative and eventually approaches zero for $T \rightarrow T_{c}$. In SmBCO the qualitative behaviour is similar, with a larger low temperature value and a much lower $T_{0}$. A deeper investigation is made by measuring the fieldsweeps at fixed temperature. In figures 3 and 4 we show typical field sweeps, $\Delta \tilde{\rho}(H) / d=[\tilde{\rho}(H, T)-\tilde{\rho}(0, T)] / d$, in YBCO and SmBCO, respectively, at temperatures chosen in both cases a few Kelvins below $T_{0}$. The data here reported are representative of the behavior observed in other $\mathrm{SmBCO}$ and $\mathrm{YBaCuO}$ films measured under the same conditions and in similar temperature and field ranges. It is seen that the field dependences of the microwave resistivity in $\mathrm{YBCO}$ and $\mathrm{SmBCO}$ are slightly 
different. In particular, in the present field range the field variation of both $\Delta \rho_{1}$ and $\Delta \rho_{2}$ in SmBCO presents a more pronounced downward curvature than in YBCO. We discuss in the following the possibile reasons for the similarities and differences between YBCO and SmBCO.

\section{DISCUSSION}

When we come to discuss the data, we are faced with the increase of the imaginary part in even moderate fields at low enough temperatures (see figures $2,3,4$ ). Since the role of pinning reveals itself mostly on the imaginary part, one might be tempted to assign the quantitative difference between YBCO and SmBCO to a different pinning, stronger in SmBCO. Following this quite conventional view, we first discuss the data in terms of simple vortex dynamics, assuming that the observed resistivity is entirely due to vortex motion (VM). To this aim, we take the simplest model for $\tilde{\rho}_{v m}$ as early proposed by Gittleman and Rosenblum ${ }^{13}$ (GR):

$$
\tilde{\rho}=\tilde{\rho}_{v m}(H, T)=\frac{\Phi_{0} B}{\eta} \frac{1+\mathrm{i} \frac{\omega_{\mathrm{p}}}{\omega}}{1+\left(\frac{\omega_{p}}{\omega}\right)^{2}}
$$

where $\eta$ is the unit length vortex viscosity, $\omega_{p}$ is the depinning angular frequency and $k_{p}=\omega_{p} \eta$ is the unit length pinning constant. This analysis has been widely used in various HTCS, see the review in Ref. 3. This model, based on the motion of independent vortices, is expected to be more and more accurate with increasing frequency: at high operating frequencies vortex displacements induced by the microwave field are much smaller than the intervortex mean spacing, and complications arising from the collective nature of the vortex matter are less and less relevant. In fact, it has been experimentally shown ${ }^{14}$ that in YBCO a dynamic crossover from a glassy behaviour to the independent vortex motion takes place above a few GHz. We note that the GR model explicitly predicts that the imaginary resistivity should increase with the application of the field. This result is common to other VM based models, based on less restrictive assumptions. It is then apparent that, at least in a temperature range of several Kelvins below $T_{c}$ (above $T_{0}$ ), the data cannot be explained on the basis of $\mathrm{VM}$ alone. At low enough temperatures, applying in a straightforward way the GR model one would get directly from the data the depinning frequency $\omega_{p} / 2 \pi$, the vortex viscosity $\eta$ and the pinning constant $k_{p}$. In figures 3 and 4 (right panels) we report the calculated vortex parameters in YBCO and SmBCO. We immediately note that in both materials the vortex viscosity and pinning constant increase with the field, while the depinning frequency increases in YBCO and is approximately constant in SmBCO. The field dependence of the soobtained $\eta$ and $k_{p}$ is not easily explained. In particular, one would expect a nearly constant vortex viscosity, as experimentally determined by multifrequency measurements at lower temperatures ${ }^{6}$ and by high-field sweptfrequency Corbino disk measurements, ${ }^{15}$ and a weak field dependence of $k_{p}$. Exotic field dependences of the vortex viscosity, increasing with the field as $\eta \sim \sqrt{B}$, have been observed in $\mathrm{Bi}_{2} \mathrm{Sr}_{2} \mathrm{CuO}_{6}$ and tentatively explained ${ }^{16}$ in terms of a different field dependence of quasiparticle relaxation time in a d-wave superconductor, appearing at high fields when the intervortex distance becomes smaller than the mean free path. This picture does not apply easily to our case, since the field range is very different. In addition, this interpretation would not give an explanation of the field increase of $k_{p}$ (we remark that the anomalies reported in Ref. 16 were confined to $\eta$ : $k_{p}$ was found constant at low fields, and decreasing at higher fields). We conclude that this explanation is at least questionable at low fields.

Granularity is sometimes indicated as a possible dominant source for the losses in the microwave response in superconducting films. Manifestations of granularity include weak-links dephasing, ${ }^{17}$ Josephson fluxon (JF) dynamics ${ }^{18}$ and, as recently studied, AbrikosovJosephson fluxon (AJF) dynamics. ${ }^{19}$ Weak-links dephasing is charaterized by a very sharp increase of the dissipation at dc fields of order or less than $20 \mathrm{mT}$, accompanied by a strong (and sometimes exceptionally strong) hysteresis with increasing or decreasing field. ${ }^{17}$ However, those effects are relevant in large-angle grain boundaries, such as those found in pellets and granular samples, and are not observed in good thin films. In fact, we did not observe none of the above mentioned effects in our films, so we conclude that weak-links dephasing does not affect our measurements. Josephson fluxon dynamics has been studied essentially in relation to nonlinear effects, due to the short JF nucleation time. If however one assumes that a dc field has the same effect as a microwave field, the qualitative properties of JF dynamics would be barely distinguishable from conventional Abrikosov fluxons dynamics, since an equation like Eq.4 would hold, ${ }^{18}$ with the noticeable difference of a larger pinning frequency (due to the small viscosity, due in its turn to the insulating core). Reactive response should then dominate, while in our measurements (see figs. 2,3,4) the dissipation $\left(\Delta \rho_{1}\right)$ is always larger than reactance $\left(\Delta \rho_{2}\right)$. We are led to conclude that JF dynamics, even if possibly present, is not the main mechanism driving the measured microwave response.

Another possibility is the role played by AbrikosovJosephson fluxons. Such flux structures nucleate along small-angle grain boundaries, as those presumably present in our oriented films. The ac response is expected to saturate at fields larger than a characteristic field $H_{0}$, whose estimate spans orders of magnitude, ${ }^{19}$ in the range 0.1-10 T. In YBCO bicrystals, dc measurements ${ }^{20}$ yielded $H_{0}<2 \mathrm{~T}$ above $T=70 \mathrm{~K}$. However, it is noteworthy that in defective thin films of other cuprates $\left(\mathrm{Tl}_{2} \mathrm{Ba}_{2} \mathrm{CaCu}_{2} \mathrm{O}_{8+x}\right)$ the $8.5 \mathrm{GHz}$ dissipation ascribed to 
AJ fluxon motion is shown to saturate at (microwave) fields as low as $\mu_{0} H_{0} \sim 1 \mathrm{mT},{ }^{21}$ while in better films this kind of response is not present at all. Nevertheless, allowing for a large $H_{0}$, the explicit expression for AJ fluxon motion complex resistivity ${ }^{19}$ yields an initial magneticfield increase in the resistivity as $\sim \sqrt{\frac{H}{H_{0}}}$, whereas the reactance initially decreases with the magnetic field as $\sim-\sqrt{\frac{H}{H_{0}}}+c(T) \frac{H}{H_{0}}$ with $-\frac{1}{2}<c<\frac{1}{2}$. It appears that our data, where the reactance increases or decreases with the field dependending on the temperature, are not easily described by this only mechanism.

Summarizing, none of the mechanisms shortly recalled above appears to describe the main qualitative features of our data, namely: (i) the sublinear variation of the real and imaginary parts of the resistivity, (ii) the change of sign of the variation of the reactance with increasing temperature. It is not excluded that a properly tailored combination of several of the above mechanisms could describe our data. Nevertheless, in order to look for a single possibly dominant mechanism in the microwave complex response to a dc magnetic field we now comment on a possible alternative explanation for our data. In fact there is quite robust evidence, in other HTCS, that in the vortex state the quasiparticle increase has a relevant role, at least in the imaginary response. ${ }^{7}$ This phenomenon is well explained in terms of a semiclassical theory of a superconductor with line of nodes in the $\mathrm{gap}^{22}$. In particular, the decrease of superfluid fraction is predicted to follow a $B^{\alpha}$ law, with $\alpha=\frac{1}{2}$ in clean d-wave superconductors. In this case the charge carrier conductivity can be described by the two-fluid model, and cast in the form:

$$
\tilde{\sigma}(H, T)=\sigma_{R 0} x_{n}(T, B)-\mathrm{i} \sigma_{I 0} x_{s}(T, B)
$$

where $x_{n}(T, B)$ and $x_{s}(T, B)=1-x_{n}(T, B)$ are the temperature and field dependent normal and superfluid fractional densities, and $x_{s}(T, B)=x_{s}(T, 0)\left[1-\left(B / B_{p b}\right)^{\alpha}\right]$ where $B_{p b}$ is a pair breaking field. It has been shown ${ }^{23}$ that in clean superconductors $B_{p b} \propto B_{c 2}(T)$, but deviations from this behaviour are possible.

As a very crude approximation, we can assume as a limiting case the vortex motion does not give a significant contribution to the resistivity. Taking into account that $\left(B / B_{p b}\right)^{\alpha} \ll 1$, one can show ${ }^{24}$ that in this case the field variation of the complex resistivity can be written as:

$$
\Delta \tilde{\rho}(H, T)=\left[a_{1}(T ; s)+\mathrm{i} a_{2}(T ; s)\right]\left(\frac{B}{B_{p b}}\right)^{\alpha}
$$

where the dependence on the single parameter $s=\frac{\sigma_{R 0}}{\sigma_{I 0}}$ has been made explicit. By considering the temperature dependent data in figure 2 , we do not need to assume any specific field dependence. However, we remark that in SmBCO the data are well described with $\alpha=\frac{1}{2}$ (Ref. ${ }^{24}$ ). Due to the oversimplification of the model, for the temperature dependences involved in Equation
6 we take conservatively the most simple: the superfluid fraction $x_{s}(T, 0)=\left(1-t^{2}\right)$, and $B_{p b} \propto B_{c 2}(T)$, so that $B_{p b}=B_{p b 0}\left(1-t^{2}\right)$, with $t=T / T_{c}$. In order to gain qualitative information, we do not attempt to insert some temperature dependence in the parameter $s$. We stress that, with this choice, $s$ is not a free parameter: its value is determined by the requirement that the calculated curve of $\Delta \rho_{2}$ changes sign at the experimental temperature $T_{o}$. The only free parameter is the scale factor given by the pair breaking field. The model is surely oversimplified, but has rather strong constraints: in particular the shapes of the two calculated curves $\Delta \rho_{1}(T)$ and $\Delta \rho_{2}(T)$ are determined, and only a common scale factor can be adjusted. The resulting calculated curves for the experimentally applied field $B_{a}=0.7 \mathrm{~T}$ are reported in figure 2. As can be seen, the overall shape of the experimental curves are reproduced. From the scale factors we estimate $\left(\frac{B_{a}}{B_{p b 0}}\right)^{\alpha} \sim 0.05$ in $\mathrm{YBCO}$ and SmBCO. One also gets $s \simeq 0.1$ and 0.2 in $\mathrm{YBCO}$ and $\mathrm{SmBCO}$, respectively. We note that by applying the conventional two-fluid model, $\tilde{\sigma}=\frac{n e^{2}}{m \omega}\left[\omega \tau x_{n}-\mathrm{i} x_{s}\right]$, one has $s=\omega \tau$ where $\tau$ is the quasiparticle scattering time. Taking into account the measuring frequency, one has $\tau \simeq 0.35 \mathrm{ps}$ and $0.7 \mathrm{ps}$ in YBCO and SmBCO, respectively, that compare well to, e.g., $0.2 \mathrm{ps}$ at $\approx 80 \mathrm{~K}$ as obtained from microwave measurements in $\mathrm{YBCO}$ crystals $^{25}$ and to $0.5 \mathrm{ps}$ at $\approx 80 \mathrm{~K}$ as obtained from millimeter-wave interferometry in YBCO film. ${ }^{26}$ We note that the model appears to describe better the data on SmBCO than on YBCO: this is in fact consistent with the much stronger curvature of the field-sweeps data in $\mathrm{SmBCO}$, since the proposed fieldinduced pair breaking mechanism is naturally sublinear in superconductors with nodes in the gap. In fact, in SmBCO the small difference between the calculated pair breaking response and the experimental data can be well described by vortex motion in the free-flow limit. ${ }^{24,27} \mathrm{In}$ this respect, the discrepancy between the calculated pairbreaking and the experimental data, as well as deviations from $H^{\frac{1}{2}}$ dependence of the field sweeps, is most likely due to motion of flux lines, either JF, AJF or Abrikosov fluxons. We believe that the successful application of the oversimplified pair-breaking model in the qualitative description of the vortex-state resistivity in $\mathrm{RE}(\mathrm{Y}, \mathrm{Sm}) \mathrm{BCO}$ points toward a substantial contribution of pair breaking by the magnetic field, in agreement with the existence of lines of nodes in the gap, while additional mechanisms, such as fluxon motion, are most probably also present.

\section{CONCLUSION}

We have presented data for the microwave resistivity in $\mathrm{REBa}_{2} \mathrm{Cu}_{3} \mathrm{O}_{7-\delta}$ thin films as a function of the temperature and magnetic field. The field-dependence of the complex resistivity exhibits quantitatively different behaviours in the two materials. The analysis in terms of vortex motion alone, in particular ascribing the quan- 
titative differences to a different pinning, would imply very exotic field dependences of the vortex parameters, so that it appears that alternative interpretations have to be found, including a possible enhanced role of the field-induced pair breaking. By ascribing, as a limiting case, the entire response to the field-induced pair-braking we obtain semi-quantitative fits of the data at $0.7 \mathrm{~T}$ as a function of the temperature, with a large quasiparticle scattering time below $T_{c}$ in agreement with estimates given by several groups. Additional measurements might be required in order to fully understand the interplay between fluxon motion and pair breaking.

\section{ACKNOWLEDGEMENTS}

Valuable assistance by L. Muzzi is warmly acknowledged. We benefited from useful discussions and remarks by M.W. Coffey, J. R. Clem, S. Anlage, D. Oates, R. Woerdenweber, J. Halbritter and A. Maeda. We thank A.M. Cucolo and M. Boffa at Università di Salerno for the SmBCO sample. This work has been partially supported by Italian MIUR under FIRB "Strutture semiconduttore/superconduttore per l'elettronica integrata".

${ }^{1}$ H.A. Blackstead, D.B. Pulling, C.A. Clough, Phys. Rev. B 47, 8978 (1993); E. Silva, R. Marcon and F.C. Matacotta, Physica C 218, 109 (1993)

${ }^{2}$ M.W. Coffey and J.R. Clem, Phys. Rev. Lett. 67, 386 (1991)

${ }^{3}$ M. Golosovsky, M. Tsindlekht, D. Davidov, Supercond. Sci. Technol. 9, 1 (1996), and references therein

${ }^{4}$ R.Marcon, R.Fastampa, M.Giura, E.Silva, Phys. Rev. B 43, 2940 (1991)

${ }^{5}$ S. Revenaz, D. E. Oates, D. Labbé-Lavigne, G. Dresselhaus, and M. S. Dresselhaus, Phys. Rev. B 50, 1178 (1994)

${ }^{6}$ Y. Tsuchiya, K. Iwaya, K. Kinoshita, T. Hanaguri, H. Kitano, A. Maeda, K. Shibata, T. Nishizaki, and N. Kobayashi, Phys. Rev. B 63, 184517 (2001).

${ }^{7}$ R. Mallozzi, J. Orenstein, J.N. Eckstein and I. Bozovic, Phys. Rev. Lett. 81, 1485 (1998).

${ }^{8}$ C. Beneduce, F. Bobba, M. Boffa, A.M. Cucolo, M.C. Cucolo, A. Andreone, C. Aruta, M. Iavarone, F. Palomba, G. Pica, M. Salluzzo, R. Vaglio, Int. J. Mod. Phys. B 13, 1333 (1999); M.A. Boffa, F. Bobba, A.M. Cucolo, R. Monaco, Int. J. Mod. Phys. B 17, 768 (2003); M. Boffa, M.C. Cucolo, R. Monaco, A.M. Cucolo, Physica C 384, 419 (2003).

${ }^{9}$ C. Camerlingo, M.P. Lissitski, M. Russo, M. Salvato, presented at INFMeeting, June 2002, Bari, Italy (unpublished)

${ }^{10}$ E. Silva, A. Lezzerini, M. Lanucara, S. Sarti and R. Marcon, Meas. Sci. Technol. 9, 275 (1998)

${ }^{11}$ E. H. Brandt, Rep. Prog. Phys. 58, 1465 (1995); C. P.
Poole, Jr., H. A. Farach, R. J. Creswick, Superconductivity, Academic Press, 1995

12 E. Silva, M. Lanucara, R. Marcon, Supercond. Sci. Technol. 9, 934 (1996); N. Pompeo, R. Marcon and E. Silva, Proceedings of 6th European Conference on Applied Superconductivity - EUCAS 2003, 14-18/9/2003, Sorrento (Italy), paper 709, to be published.

13 J. I. Gittleman and B. Rosenblum, Phys. Rev. Lett. 16, 734 (1966)

${ }^{14}$ D. H. Wu, J. C. Booth, and S. M. Anlage, Phys. Rev. Lett. 75, 525 (1995).

15 S. Sarti, E. Silva, C. Amabile, R.Fastampa, M.Giura, Physica $C$ 404, 330 (2004)

${ }^{16}$ Y. Matsuda, A. Shibata, K. Izawa, H. Ikuta, M. Hasegawa, Y. Kato, Phys. Rev. B 66, 014527 (2002)

${ }^{17}$ R. Marcon, R. Fastampa, M. Giura, C. Matacotta, Phys. Rev. B 39, 2796 (1989); M. Giura, R. Marcon, R. Fastampa, Phys. Rev. B 40, 4437 (1989); M. Giura, R. Fastampa, R. Marcon, E. Silva, Phys. Rev. B 42, 6228 (1990);

18 J. Halbritter, J. Supercond. 8, 691 (1995)

${ }^{19}$ A. Gurevich, Phys. Rev. B 46, 3187 (1992); Phys. Rev. B 65, 214531 (2002)

${ }^{20}$ A. Gurevich, M.S. Rzchowski, G. Daniels, S. Patnaik, B.M. Hinaus, F. Carillo, F. Tafuri, D.C. Larbalestier, Phys. Rev. Lett. 88, 097001 (2002)

${ }^{21}$ E. Gaganidze, R. Heidinger, J. Halbritter, A. Shevchun, M. Trunin, H. Schneidewind, J. Appl. Phys. 93, 4049 (2003)

${ }^{22}$ G.E. Volovik, JETP Lett. 58, 469 (1993)

${ }^{23}$ H. Won and K. Maki, Phys. Rev. B 53, 5927 (1996).

${ }^{24}$ E. Silva, N. Pompeo, L. Muzzi, R. Marcon, S. Sarti, M. Boffa, A.M. Cucolo, submitted for publication (2004), cond-mat/0405324

${ }^{25}$ D.A. Bonn, R. Liang, T.M. Riseman, D.J. Baar, D.C. Morgan, K. Zhang, P. Dosanjh, T.L. Duty, A. MacFarlane, G.D. Morris, J.H. Brewer, W.N. Hardy, C. Kallin, A.J. Berlinsky, Phys. Rev. B 47, 11314 (1993).

${ }^{26}$ T. Nagashima, M Hangyo, S. Nakashima, Y Murakami, Adv. in Superconductivity VI, ed. by T. Fujita and Y Shiohara, Springer-Verlag Tokyo 1994, pp. 209-212

${ }^{27}$ E. Silva, R. Marcon, L. Muzzi, N. Pompeo, R. Fastampa, M. Giura, S. Sarti, M. Boffa, A.M. Cucolo, M.C. Cucolo, Physica C 404, 350 (2004) 

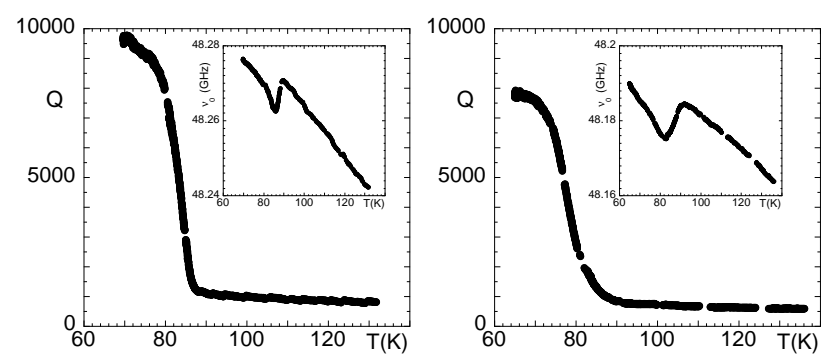

FIG. 1. Temperature dependence of the coeffcients of the resonant cavity incorporating as an end wall the superconducting film. Main panels, quality factors; insets, resonant frequency. Left panel: YBCO film; right panel, SmBCO film.
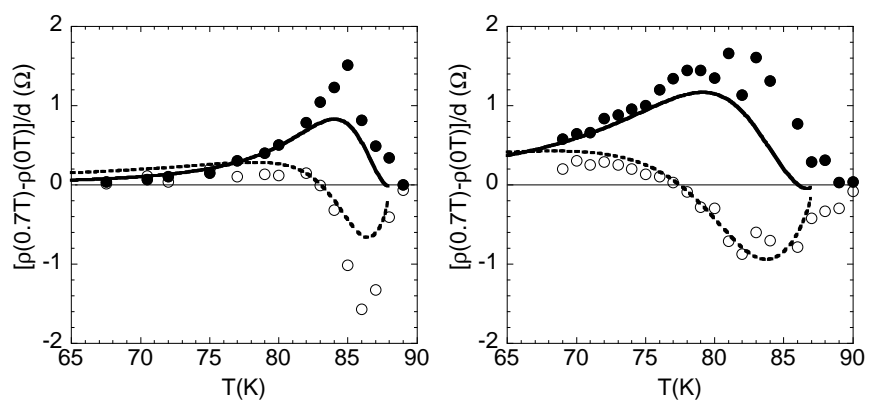

FIG. 2. Variation of the resistivity with the application of a $0.7 \mathrm{~T}$ magnetic field. Left panel reports data on YBCO, right panel in SmBCO. Full symbols: shift of the real part, $\left[\rho_{1}(T, 0.7 \mathrm{~T})-\rho_{1}(T, 0)\right] / d$. Open circles: shift in the imaginary part, $\left[\rho_{2}(T, 0.7 \mathrm{~T})-\rho_{2}(T, 0)\right] / d$. Lines are calculated curves with Eq. 6 with a common scale factor as the only free parameter.
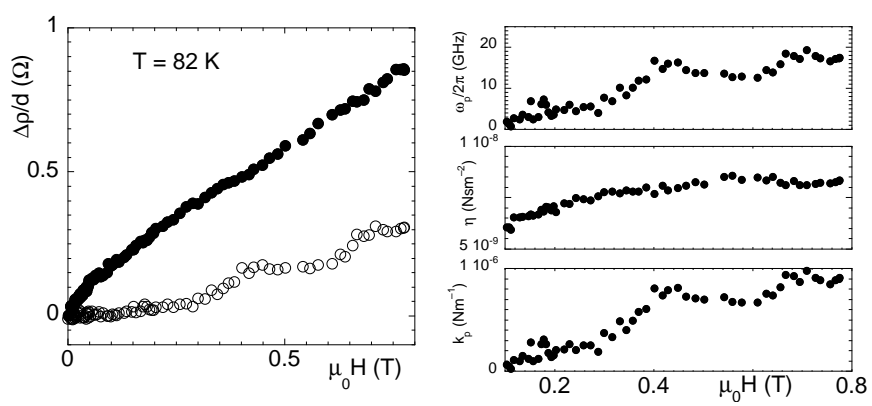

FIG. 3. Left panel: field induced change in the real (full circles) and imaginary (open circles) part of the complex resistivity. Right panels: vortex parameters as calculated from data in YBCO accordingly to the conventional Gittleman-Rosenblum model. From top to bottom: depinning frequency $\omega_{p} / 2 \pi$, unit length vortex viscosity $\eta$, unit length pinning constant $k_{p}$. The strong field dependence of the so-calculated vortex viscosity and pinning constant cannot be easily justified.
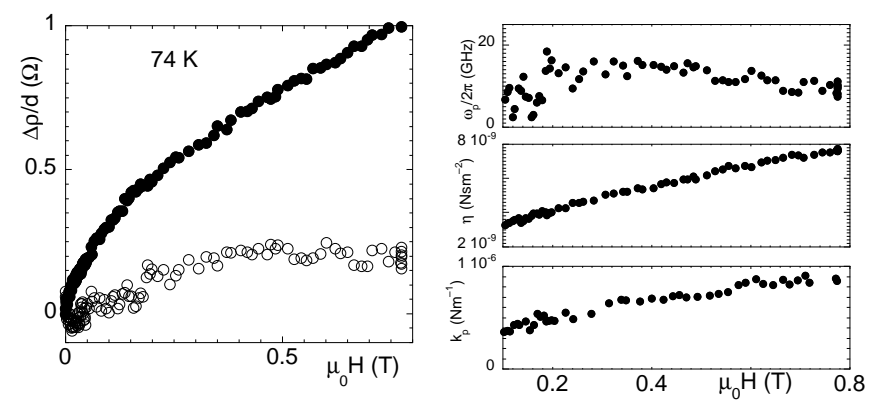

FIG. 4. Same as in Figure 3, but for the SmBCO film. 